\title{
Diagnostic Dilemma: Neurocysticercosis-A Differential Diagnosis for Seizures in Pregnancy and Early Postpartum Period
}

\author{
Shilpi Singh ${ }^{1}$, Munikrishna $\mathrm{M}^{2}$
}

\begin{abstract}
The most common helminthic infestation of the brain worldwide is neurocysticercosis (NCC). When a woman presents with seizures during pregnancy, eclampsia is the go to provisional diagnosis. NCC may be misdiagnosed because of its symptoms like headache, vomiting and nausea, neurological and visual disturbances, and seizures. Hence, radiological findings help to diagnose NCC. Treatment is with antiepileptics and antihelminthic agents.

We reported the cases of three Indian women in our tertiary care center, aged 19, 20, and 22 years, respectively, with NCC, one of whom presented with seizures in the first trimester of pregnancy and other two during the postpartum period. All patients were normotensive with urine albumin nil and no history of increased blood pressure in the past. The confirmation was done on the basis of MRI brain, which showed spherical ring-enhancing lesions that were calcified in the brain. In the first patient aged 19 years, pregnancy was terminated on patient request and she was discharged on albendazole and levetiracetam. The second and the third patients whose antenatal period was uneventful with postpartum convulsions were also put on levetiracetam and albendazole for 21 days.

Keywords: MRI brain, Neurocysticercosis (NCC), Postpartum convulsion.

Journal of South Asian Federation of Obstetrics and Gynaecology (2021): 10.5005/jp-journals-10006-1873
\end{abstract}

\section{INTRODUCTION}

Eclampsia, i.e., (Greek, "shining forth") an acute and life-threatening complication of pregnancy, is characterized by the appearance of tonic-clonic seizures in a patient of preeclampsia with maternal mortality ranging from 2 to $20 \%$; seizures in pregnancy result due to epilepsy, cerebral venous thrombosis, meningitis, encephalitis, tuberculoma, neurocysticercosis (NCC), and other CNS disorders.

$\mathrm{NCC}$, though, is a rare entity, but an important cause of seizures in pregnancy for the first time. ${ }^{2}$

It is unwise to diagnose eclampsia in pregnant women who present with seizures in early or postpartum period as other pathological causes may also be responsible as mentioned above.

There might arise difficulty in diagnosis and treatment due to overlap between symptoms. ${ }^{3}$

Sometimes we miss the diagnosis as expensive neuroimaging has limited role in uncomplicated cases with typical clinical course and prompt response to standard therapy. ${ }^{4}$ Noninvasive imaging technique such as MRI brain is not only safe in pregnancy but also a good investigative tool in aiding the diagnosis.

"Biological marker" of the social and economic development of a community is none other than cysticercosis. The most common parasitic infection of CNS is NCC. ${ }^{5}$

It is endemic in developing regions like sub-Saharan Africa, South America, and areas of Asia, specifically India, regions of SouthEast Asia, and China. In UK, the prevalence rate of NCC remains low and cases reported might be among immigrants.

When humans become intermediate hosts, cysticercosis develops through ingesting embryonated eggs of tapeworm which enter the bloodstream and release oncospheres, developing cystercerci in tissues. In the CNS, Taenia solium larvae remain dormant for several years before degenerating into granulomas and developing into calcified lesions. The infection is no longer
1,2Department of Obstetrics and Gynecology, Sri Devaraj Urs Medical College, Kolar, Karnataka, India

Corresponding Author: Shilpi Singh, Department of Obstetrics and Gynecology, Sri Devaraj Urs Medical College, Kolar, Karnataka, India, e-mail: shilpi_dudu@yahoo.co.in

How to cite this article: Singh S, Munikrishna M. Diagnostic Dilemma: Neurocysticercosis-A Differential Diagnosis for Seizures in Pregnancy and Early Postpartum Period. J South Asian Feder Obst Gynae 2021;13(2):84-86.

Source of support: Nil

Conflict of interest: None

considered active at this stage. ${ }^{6}$ But transient perilesional edema around calcified foci is common and associated with episodic seizure activity.

NCC may be misdiagnosed because of its symptoms like headache, vomiting and nausea, neurological and visual disturbances, and seizures.

Diagnostic criteria have been proposed for NCC, which are quite complex and have not been universally accepted. One of the major drawbacks is that they do not help to differentiate NCC from tuberculoma, which is also common brain imaging finding in our country.

Other modalities of investigations such as serum antibodies to T. solium by EITB (enzyme-linked immune electrotransfer blot) have also been used with a sensitivity of $98 \%$ in cases with more than one parenchymal cyst or subarachnoid disease; however, sensitivity falls to 50 to $60 \%$ in cases with only one cyst and is also poor in cases with calcified cysts. To detect anticysticercal antibodies, CSF can be done by using ELISA, if EITB is unavailable. ${ }^{5}$ Del Brutto has proposed

( ) Jaypee Brothers Medical Publishers. 2021 Open Access This article is distributed under the terms of the Creative Commons Attribution 4.0 International License (https://creativecommons.org/licenses/by-nc/4.0/), which permits unrestricted use, distribution, and non-commercial reproduction in any medium, provided you give appropriate credit to the original author(s) and the source, provide a link to the Creative Commons license, and indicate if changes were made. The Creative Commons Public Domain Dedication waiver (http://creativecommons.org/publicdomain/zero/1.0/) applies to the data made available in this article, unless otherwise stated. 
diagnostic criteria for NCC histological demonstration of parasite, clinical symptoms, serology, neuroimaging, and epidemiology. ${ }^{2}$

\section{Case Series \\ Case 1}

A 19 years old woman referred from primary health care (PHC) presented with seizures lasting for 4-5 minutes, following which she was in post ictal drowsy and disoriented state. When she reached hospital, it was roughly 6 to 8 hours after her last seizure. Patient gave a history of headache in the frontal region and fever on and off since 6 months. There was no past history of increased blood pressure readings, no history of vomiting, and no history of pedal edema, and patient was not taking any medications. There was no history of any other medical illness and nonvegetarian by diet. On examination, her pulse was 90 beats per minute, regular blood pressure was $130 / 90 \mathrm{~mm} \mathrm{Hg}$, and respiratory rate was $22 \mathrm{cpm}$. Her urine albumin with dipstick method was nil, and the liver function, coagulation profile, renal function, and fundus examinations were within normal limits. An obstetric ultrasound showed a single live intrauterine gestation of 12 weeks. An emergency MRI of our patients was advised, which showed a 3-mm spherical ring-enhancing lesion in the frontal region of her brain. Once tuberculosis was ruled out as a differential diagnosis along with other space-occupying lesion of brain, the diagnosis of NCC was made.

She was managed with intravenous levetiracetam $800 \mathrm{mg}$ twice daily for 5 days for seizures. Patient insisted on termination due to social and superstitious reasons, and hence following termination with misoprostol induction after taking informed and written consent, she was started on tablet albendazole $400 \mathrm{mg}$ twice daily for 21 days and tablet levipil 500 mg twice daily with tablet folic acid $5 \mathrm{mg}$ once a day and asked to come for a follow-up.

\section{Case 2}

A 20-year-old para 2 live 1 dead 1 [P2L1D1], booked case, post-LSCS for severe oligohydramnios and previous LSCS on day 20, presented in labor room with history of headache and fever since 7 days. She presented with history of four episodes of convulsions, generalized tonic-clonic seizures (GTCS) as described by patient attenders (not reliable). She came to our hospital on day 20 with generalized tonic-clonic seizures preceded by nausea and headache. On further enquiry with patient attenders, it was uncovered that patient had history of fever on and off for a week. However, there was no history of tinnitus or neck rigidity or visual disturbances or no history of increased blood pressure in the past, no history of pedal edema or blurring of vision. There was no history of any medical disorders, and patient had a mixed diet.

On examination, Glasgow Coma Scale (GCS) was 15/15 with higher functions being normal. Reflexes were normal and bilateral reactive pupils. She was afebrile with a temperature of $97.6^{\circ} \mathrm{F}$, pulse rate of 90 beats/minute, regular, blood pressure of $108 / 66 \mathrm{~mm} \mathrm{Hg}$, respiratory rate of $26 \mathrm{cpm}$, and $\mathrm{SpO}_{2}$ of $98 \%$ at room air. Chest examination respiratory system was normal with bilateral normal vesicular sound heard, no added sounds. Per abdomen, soft uterus was not palpable and local examination of lochia was healthy. A provisional diagnosis of atypical eclampsia was made, but she had four episodes of seizures consecutively with interseizure period in post-ictal stage when patient has not regained consciousness. All four episodes as described by patient attenders appeared to be off the GTCS variety following which she was brought into our casualty. Physician's advice was taken on an emergency basis to rule out other causes of seizures. Then, routine and specialized (blood urea and serum creatinine, electrolytes, normal coagulation profile including PT, aPTT, international normalized ratio, liver function tests, uric acid) laboratory investigations were done. Routine investigations showed a hemoglobin of $8.8 \mathrm{gm} / \mathrm{dL}$, platelets of 4.32 lakhs/cumm, and white cell count (TLC) of 13,200 cells/cumm, with normal specialized investigations. MRI scan was done, which revealed multiple ring-enhancing lesions in left high parietal lobe cortex and left midbrain, a calcified granulomatous lesion, which was diagnostic of NCC.

Then, patient was treated with inj levipil $800 \mathrm{mg}$ IV for 3 days and later shifted to oral medication, tablet albendazole for 21 days and tablet prednisolone for 5 days with tapering dose.

\section{Case 3}

An outside booked case, 22 years old P3L3 with previous 3 Lower Segment Ceserean Section (LSCS) with last child birth 6 hours prior to admission post-LSCS for previous 2 LSCS in latent labor, was referred from peripheral hospital to our tertiary care center with diagnosis of postpartum eclampsia. Patient had one episode of generalized tonic-clonic seizure on table immediately after her LSCS lasting for 3 minutes, inj fortwin $30 \mathrm{mg}$ and inj midazolam $1 \mathrm{mg}$ was given along with $4 \mathrm{gm} \mathrm{MgSO}_{4}$ loading dose according to zuspan regime.

Patient gave a history of blurring of vision in post-ictal phase. There was no history of increased blood pressure, fever, headache, vomiting, and pedal edema in the past.

There was no significant past medical/surgical or family history, and patients followed nonvegetarian diet.

On admission, examination, vitals Glasgow Coma Scale score was $14 / 15$. Her pulse rate was $76 /$ minute, regular BP was $100 / 60 \mathrm{~mm} \mathrm{Hg}$, and respiratory rate was $22 \mathrm{cpm}$. Neurological examination was within normal limits. Her fundus examination was normal. Urine albumin was nil; liver function test, renal function test, and coagulation profile were normal within the range. On abdominal examination uterus was well contracted and retracted well. Local examination was minimal bleeding through os present.

Ophthalmologist advice and physician advice were taken and followed for blurring of vision. As all the above-mentioned investigations for hypertensive disorder in pregnancy were within the normal limits, further investigations were done to rule out seizure during pregnancy and reported as follows: multiple healed calcified granulomas in subcortical region of bilateral cerebral hemispheres and right thalamus suggestive of NCC.

She was put on albendazole $400 \mathrm{mg} \mathrm{BD}$ and tablet levetiracetam $500 \mathrm{mg} \mathrm{BD}$ under cover of injection dexamethasone $4 \mathrm{mg}$ BD in first 5 days and was discharged on tablet albendazole $400 \mathrm{mg} \mathrm{BD}$ for 21 days and tablet levetiracetam $500 \mathrm{mg} \mathrm{BD}$ and asked to come for follow-up after 3 weeks.

\section{Discussion}

In developing countries, convulsions are the most common cause of manifestations of brain abnormalities caused by $T$. solium cysticercosis. The condition is caused by pork tapeworm $T$. solium-encysted larval stage that is cysticercus cellulose. The final host is man, but may sometimes become the intermediate host, by ingesting the ova shed in the feces of a human carrier, and hence, it may occur in people who do not even eat pork and have no contact with pigs. Through autoinfection, cysticercosis may develop in tapeworm carriers. Neurological sequelae followed 
by subsequent larvae settling in the brain and more rarely the spinal cord may occur. ${ }^{6}$

A thorough evaluation is crucial to differentiate NCC from other causes of seizures during pregnancy, mainly eclampsia. Neuroimaging is the definitive modality for clinching the diagnosis of NCC. The most common radiological finding in NCC is calcified T. solium cysts, which are commonly foci of seizure activity and present in 10 to $20 \%$ of the endemic population.

A study conducted in Peru showed that $50 \%$ of patients with calcified cysts and a positive cysticercosis serology presenting with recurring seizures showed perilesional edema, suggestive of NCC. ${ }^{8}$ Therefore, the use of immunosuppressive or anti-inflammatory medications, alongside antiseizure drugs in managing these patients, is highly recommended. The pathophysiology of perilesional edema is still inconclusive, yet a few hypotheses suggest the intermittent release or recognition of parasite antigen by the host or periodic loss of immune suppression results in an inflammatory reaction, is one hypothesis. ${ }^{9}$ Another theory suggests selective calcified lesions have a propensity to cause perilesional edema through the disruption of the blood-brain barrier. $^{8}$

Praziquantel and albendazole are the two anticysticercidal drugs, which are widely used in endemic area. Antihelminthic therapy decreases the burden of parasites and is safe and effective, at least in reducing seizures and generalization, in patients with seizures due to viable cysts. ${ }^{6}$ Antihelminthic treatment should be delayed in patients till postpartum patients they are neurologically stable as data regarding medications are limited and medications are still not FDA-approved. ${ }^{10}$ However, if they present with multiple episodes of seizures, life-saving medications must be administered irrespective of the status of fetus. Patients receiving these drugs should receive corticosteroids during treatment as these drugs produce an inflammatory response.

\section{ConcLusion}

Pregnant women hailing from tropical countries presenting from seizures disorder for the first time during or immediately after pregnancy after ruling out eclampsia must undergo thorough investigations to arrive at the appropriate diagnosis. A multidisciplinary approach to tackling the issue of arriving at a definite diagnosis should be undertaken by collaborating with
Department of Medicine and Department of Radiodiagnosis to successfully diagnose NCC.

\section{Ethical Approval}

Approved.

\section{Consent}

Written informed consent was obtained from the patients for publication of this case series and the accompanying images.

\section{References}

1. Patel RD, Mishra I, Pandya NC. An unusual case of intercurrent eclampsia. J South Asian Feder Obst Gynae 2014;6(1):39-40. DOI: 10.5005/jp-journals-10006-1266.

2. Del Brutto OH. Neurocysticercosis a review. Sci World J 2012:1-8. DOI: $10.1100 / 2012 / 159821$.

3. Jamadarkhana S, Law RC. Seizures in the early post-partum period: a diagnostic dilemma. Indian J Anaesth 2012;56(2):183-185. DOI: 10.4103/0019-5049.96339.

4. Kokila M, Dwivedi AD. Correlation of clinical and neuroimaging findings affecting management in postpartum eclampsia: a prospective study. J South Asian Feder Obs Gynae 2011;3(3): 125-130. DOI:_10.5005/jp-journals-10006-1147.

5. Kaur A, Kaur B, Kaur MM, Manoj M. Neurocysticercosis misdiagnosed in pregnancy as eclampsia: outcome and management. Ann Woman Child Health 2016;2(3):32-36.

6. Garcia H, Nash T, Del Brutto O. Clinical symptoms, diagnosis, and treatment of neurocysticercosis. Lancet Neurol 2014;13(12):1202-1215. DOI: 10.1016/S1474-4422(14)70094-8.

7. Escobar A. The pathology of cysticercosis. In: Palacios E, editor. Cysticercosis of the central nervous system. Springfield, IL: Charles C. Thomas 1983: pp. 27-54.

8. Nash T, Pretell EJ, Lescano AG, Bustos JA, Gilman RH, Gonzalez AE, et al. Perilesional brain oedema and seizure activity in patients with calcified neurocysticercosis: a prospective cohort and nested casecontrol study. Lancet Neurol 2008;7(12):1099-1105. DOI: 10.1016/ S1474-4422(08)70243-6.

9. Nash T. Edema surrounding calcified intracranial cysticerci: clinical manifestations, natural history and treatment. Pathogens Global Health 2012;106(5):275-279. DOI: 10.1179/2047773212Y.0000000026.

10. D Cruz RF, Ng SM, Dassan P. Case report. Neurocysticercosis in pregnancy: maternal and fetal outcomes. OMCR 2016;2016(7):138140. DOI: $10.1093 / o m c r / o m w 019$. 